Acta Manilana 64 (2016), pp. 17-23

Printed in the Philippines

ISSN: 0065-1370

\title{
Comparison of cellular-based viability and apoptosis assays on doxorubicin treated colorectal adenocarcinoma cells
}

\author{
Thomas Adrian Tiongson ${ }^{1}$, Maria Charina Magsumbol, \\ Mark Kevin Devanadera ${ }^{1,2,3}$, \& Myla R. Santiago ${ }^{* 1,2,3}$ \\ ${ }^{1}$ Department of Biochemistry, Faculty of Pharmacy, ${ }^{2}$ Research Center for Natural and Applied Sciences, \\ ${ }^{3}$ The Graduate School, University of Santo Tomas, 1015 Manila, Philippines
}

There are various assays that can be performed to measure cell viability and apoptosis, which is why in this study, comparison of some common assays, MTT and resazurin reduction assays for cell viability and, Flow cytometry and caspase 3/7 for cell apoptosis, were performed in Doxorubicin (DOX) treated colorectal adenocarcinoma cells. These cellbased assays were analyzed in order to determine which of the paired methods are more efficient in measuring cell viability and apoptosis at various concentrations of DOX and varied incubation times. Results showed that incubation time has no effect on both cell viability assays (MTT and resazurin). Resazurin was observed to be more sensitive than MTT in measuring cell viability while MTT is more specific in determining cytotoxicity. In terms of cell apoptosis, caspase 3/7 is more specific and sensitive because of its enzyme substrate reaction. However, annexin V was more sensitive than caspase $3 / 7$ in detecting early apoptosis, late apoptosis and discrimination of necrotic cell from apoptotic cell in DOX-treated colorectal adenocarcinoma cells. Depending on application, cellular based assays for both viability and apoptosis have to be routinely standardized as different factors may affect its result such as culture mediums, buffers, cell density, $\mathrm{pH}$, incubation temperature, humidity, chemical constituents, drug dosage, and incubation time.

Keywords: MTT, resazurin, flow cytometry, caspase 3/7, luminescence assay

\section{INTRODUCTION}

Cell viability and cytotoxicity assays are being often used in cytotoxicity test of chemicals being acted upon the cell culture.

Cell viability assays measure the number of healthy cells in a sample and indicate the extent

*To whom correspondence should be addressed mrsantiago@ust.edu.ph of cell proliferation in a population. It is useful in the assessment of the potency of cytotoxic drugs, such as anticancer agents. Measurements of cell viability can be used to correlate cell number and cell behavior [1]. There are various techniques used for the detection of cell viability, based on many cell functions including, cell adherence, enzyme activity, permeability of the cell membrane, etc. Among the commonly used procedures are the MTT 
assay and the resazurin (Alamar blue) assay. These methods are based on the change in the color of the reagent dye due to mitochondrial enzymes excreted by living cells [8-11].

Cell apoptosis is best characterized as a morphological distinct form of cell death. Apoptosis, ever since, has been recognized as a distinctive mode of "programmed" cell death, in which elimination of cells is genetically determined. This event occurs normally as a homeostatic mechanism during development and aging for the sole purpose of maintenance of cell populations in tissues. Also, apoptosis can occur as a defense mechanism such as in immune reactions and when a disease damages the cells [3]. Several methodologies have been applied for the detection of apoptosis based on the various events accompanying programmed cell death. Among the common techniques are caspase analysis which detects the release of active caspase enzymes through a luminescence method and flow cytometry analysis based on the detection of alteration in the membrane of apoptotic cells through the staining dye annex in $\mathrm{V}$.

In this study, two cell viability assays (MTT and resazurin) were paired and two apoptosis assays (flow cytometry and caspase 3/7 luminescence) were paired to compare the sensitivity and specificity of these methods in measuring cell viability and apoptosis in colorectal adenocarcinoma cells treated with doxorubicin (DOX). These cell-based assays were analyzed in order to determine which of the methods are more efficient in measuring cell viability and apoptosis at various concentrations of DOX and varied incubation times. Sensitivity and tumor-resistance against cytotoxic drug (DOX) is an important factor on determining the effectivity of a certain cytotoxic drug and its effect on a certain cell line (colorectal adenocarcinoma).
Doxorubicin, also known as doxorubicin hydrochloride, is a chemotherapeutic drug used in treatment of numerous cancers, like breast, ovarian, bladder, etc. [4]. It inhibits the growth of solid tumors. This drug belongs to a class of medications called anthracycline antibiotics made from the fungus Streptomycin. In chemotherapy, one of the most widely-used drugs for the treatment of colorectal cancer is DOX.

\section{EXPERIMENTAL}

Cell culture. Colorectal adenocarcinoma cells were donated by Globetek Science Foundation and were cultured on a tissue culture flask using Minimum Essential Media (MEM) with Fetal Bovine Serum (FBS) and antibiotic-antimycotic. The flask containing the cells were incubated at $37^{\circ} \mathrm{C}$ humidified incubator with $5 \% \mathrm{CO}_{2}$. The cells were viewed in a phase contrast microscope and observed for $90 \%$ confluence and complete attachment of cells prior to assay.

Sample treatment. Cells from the flask were trypsinized using trypsin-EDTA solution and the detached cells were centrifuged. Cell pellets were collected and mixed with supplemented MEM. A cell suspension in MEM was mixed with trypan blue and the mixture was placed in a hemacytometer for cell counting. Prior to seeding all of samples were done in triplicates. The counted cells were diluted, seeded into a 96well plate, and incubated for $24 \mathrm{~h}$ prior to assay. After seeding, the cells were treated with Doxorubicin $\mathrm{HCl}$ (DOX) with $50 \mu \mathrm{g} / \mathrm{mL}, 25 \mu \mathrm{g} /$ $\mathrm{mL}, 12.5 \mu \mathrm{g} / \mathrm{mL}$, and $6.25 \mu \mathrm{g} / \mathrm{mL}$ and were incubated for $24 \mathrm{~h}$ prior to assay.

MTT assay. The cells treated with DOX at different concentrations and untreated cells were mixed with $5 \mathrm{mg} / \mathrm{mL}$ MTT solution (3-(4,5dimethylthiazol-2-yl)-2,5-diphenyl tetrazolium bromide) [1]. The wells treated with MTT dye were incubated for $4 \mathrm{~h}$ and sterilized DMSO was introduced to dissolve formazan crystals and 
mixed well. Absorbance was measured at $570 \mathrm{~nm}$ for MTT using a Corona Microplate reader SH1000 (Hitachi, Japan) [9]. After 24 h of incubation at room temperature, the absorbance of the 96well plate was measured and compared with results with 4-hour incubation.

Resazurin reduction assay. Cells treated with DOX and untreated cells were mixed with $0.15 \mathrm{mg} / \mathrm{mL}$ concentration of resazurin that has been dissolved in Dulbecco's Phosphate Buffer Saline (DPBS) with pH 7.4 and filter sterilized. The 96-well plate containing the cells and dye was incubated for 4 and $24 \mathrm{~h}$ at $37^{\circ} \mathrm{C}$ in a humidified incubator with $5 \% \mathrm{CO}_{2}$. [7]. Absorbance was read at $560 \mathrm{~nm}$ using a Corona Microplate reader SH-1000 (Hitachi, Japan).

Flow Cytometry analysis using Annexin V. Muse $^{\mathrm{TM}}$ Annexin V \& Dead Cell Kit (Merck Millipore, Germany) was used for the apoptotic cell assay following the manufacturer's protocol. Flow cytometry is used to analyze the physical and chemical characteristics of particles in a fluid as it passes through at least one laser. Fluorescently labelled cell components are excited by the laser to emit light at varying wavelengths. A 24-h cultured treated and untreated cells were placed in a microcentrifuge tubes and mixed with Muse ${ }^{\mathrm{TM}}$ Annexin V \& Dead Cell Reagent (Merck Millipore, Germany). The samples with the reagent were mixed, placed in dark, and incubated at room temperature for $20 \mathrm{~min}$. After incubation, samples were introduced to the flow cytometer (Muse ${ }^{\mathrm{TM}}$ Cell Analyzer, Merck Millipore, Germany). The data from the flow cytometer were gathered and analyzed.

Caspase 3/7 analysis using luminescence. Luminescence caspase assay is used to determine quantitatively cell apoptosis. Caspases helps stimulate the cascade of signaling events that activates cell death. Cell health assay using Caspase Glo ${ }^{\mathrm{TM}}$ 3/7 for apoptosis screening of the drug on the colorectal cancer cells was done based on the manufacturer's protocol (Promega, USA). Caspase-Glo ${ }^{\mathrm{TM}}$ 3/7 Reagent was mixed with equal volume of Caspase-Glo ${ }^{\mathrm{TM}}$ 3/7 Buffer. Blank sample which contain the reagent and the cell culture medium, the negative control which constitute the reagent, cell culture medium with cancer cells, and the test sample which composed of the reagent, cell culture medium with treated cancer cells. Caspase-Glo ${ }^{\mathrm{TM}}$ 3/7 was mixed equally with the cell culture medium containing the untreated and treated cancer cells; it was mixed at $300 \mathrm{rpm}$ for $30 \mathrm{~s}$ and incubated at room temperature for $3 \mathrm{~h}$. After incubation, the mixtures were read using Promega Luminometer and caspase activity or apoptosis rate was measured in terms of Relative Light Unit (RLU).

To compute for apoptotic rate of untreated cells, $\%$ apoptosis $=($ RLU of untreated $/$ cell concentration $\times 100$; while for cells treated with DOX, \% apoptosis $=([$ RLU of treated cells RLU of untreated cells] / RLU of treated cells) $\times$ 100.

\section{Results AND DISCUSSION}

Cellular-based viability assay. The colorectal adenocarcinoma cells were viewed under a phase contrast microscope before and after its treatment with DOX. It was observed that the cancer cells had undergone lysis after the treatment with the DOX (Fig. 1). Cellular lysis

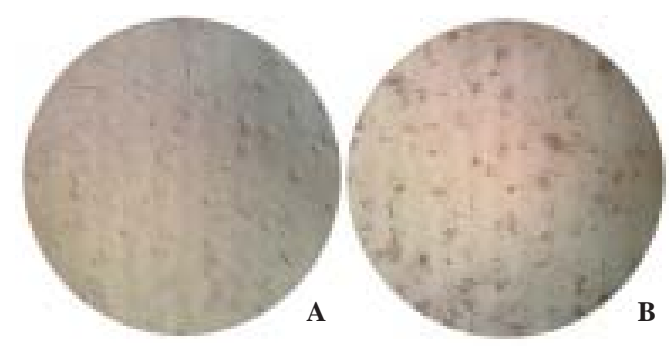

Figure 1. Micrograph analysis of (A) untreated colorectal adenocarcinoma cells and (B) doxorubicin treated colorectal adenocarcinoma cells. 
was observed as small particles present on the culture plate with orange coloration because of the orange color absorb by the cell from the DOX. Figure 1 only shows that DOX is very toxic even at low concentration on colorectal adenocarcinoma cells.

In vitro cytotoxicity assay of colorectal adenocarcinoma cells was done by treating it with various concentrations of DOX (6.25-50 $\mathrm{\mu g} /$ $\mathrm{mL}$ ) for $24 \mathrm{~h}$. Inhibition of cell viability was measured using MTT and resazurin reduction assay at 24 and $48 \mathrm{~h}$ incubation. As seen in Fig. 2, each concentrations of DOX have inhibition greater than $50 \%$. Therefore, the $\mathrm{IC}_{50}$ of DOX was noted to be less than $6.25 \mu \mathrm{g} / \mathrm{mL}$.

In addition, as DOX concentration increases, the inhibition of the drug against the viability of the colorectal adenocarcinoma cells increases for both parameters of incubation time (Fig. 2). Incubation time of 4 and $24 \mathrm{~h}$ resulted no significant difference $(p>0.05)$ for both MTT and resazurin cytotoxicity assay (Table 1 ) using paired t-test. This indicates that incubation time has no effect on measuring of cell viability though both assays have relatively similar results, but on the lowest concentration, resazurin assay showed a sudden increase on percentage inhibition (Fig. 2). Sudden increase on the percent inhibition using colorimetric

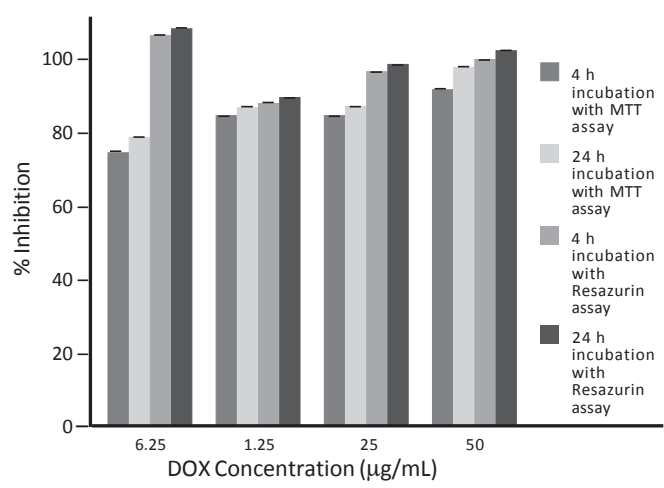

Figure 2. Comparison of cytotoxicity assay (MTT vs. resazurin) of colorectal adenocarcinoma cells against free DOX at different concentrations and incubation times. reaction may be due to the sensitivity of the dye to react on viable cells and with that sudden change on the percentage inhibition with respect to the MTT assay, it can be noted that the resazurin dye is more sensitive to other factors than the MTT dye.

Although resazurin reduction assay was observed to be more sensitive than MTT, the lowest concentration of DOX exhibited an irregular low inhibition rate (Fig. 2). According to some protocols, exposing cells to resazurin for several hours or days changes the cell morphology but does not exhibit toxic effect on the cells suggesting interference with normal cell function. The cell exposure to this dye possibly depletes reduced forms of nucleotides resulting in other effect rather than cytotoxic effects [7]. A comparison on the reaction of metabolic indicators such as MTT and resazurin has been analyzed and it was noted that the MTT dye can be reduced by NADPH, FADH, FMNH, and NADH but not with cytochrome c which is inversely proportional to the resazurin, in which it reacts with other metabolic reaction products including cytochromes to monitor all the reducing environment within a living system [12].

Statistical analysis of both colorimetric assay used paired $t$-test to analyze the differences between the two assay procedures. There are drastic differences between the assays in both the $4 \mathrm{~h}$ and $24 \mathrm{~h}$ incubation of MTT and resazurin assays shown in Fig. 2. It has been noted that incubation time of each dye has a

Table 1. Comparison of Paired $t$-Test of MTT and Resazurin Assay

\begin{tabular}{|c|c|c|c|}
\hline \multicolumn{4}{|c|}{ Paired Samples Correlations } \\
\hline & & Correlation & Sig. \\
\hline Pair 1 & MTT4 \& MTT24 & .974 & .026 \\
\hline Pair 2 & RES4 \& RES24 & .999 & .001 \\
\hline Pair 3 & MTT4 \& RES4 & -.425 & .575 \\
\hline Pair 4 & MTT24 \& RES24 & -.204 & .796 \\
\hline
\end{tabular}


great correlation and significant within the group assay but are not correlated and are insignificant between MTT assay and resazurin assay regardless of the incubation time as shown in Table 1.

In cytotoxicity assays, the following parameters have to be greatly considered such as assay procedure, cell line to be tested and the endpoint determination wherein the correlation between measurements, limitation of the assay procedure and cell viability are taken into considerations $[8,12,14]$. Assays to be used in cytotoxicity are very sensitive on cell type like cell lines or primary cells, adherent or floating cells, and sometimes depending on the cell morphology, cycle and metabolism. These bioassays may be affected by different factors such as culture mediums, buffers, cell density, $\mathrm{pH}$, micro-titer plate, incubation temperature, humidity, chemical constituents, drug dosage, and incubation time [10, 12].

Based on the results, both MTT assay and resazurin assay are sensitive in terms of assay procedure and its reaction to the metabolic

A
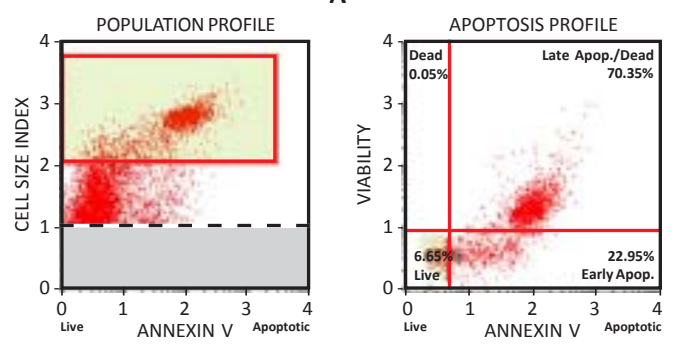

B
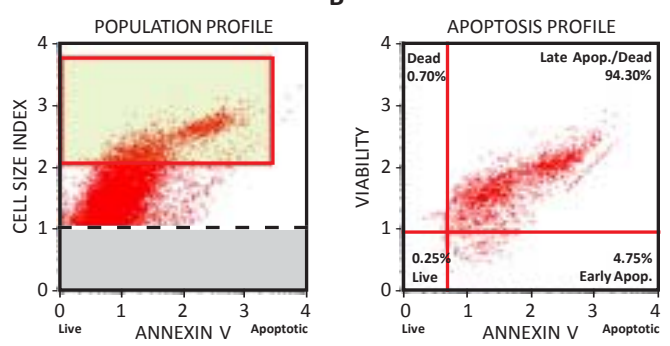

impairment reaction. However, MTT is more specific in determining cytotoxicity since it only reacts on certain metabolic products and is not affected by other reducing agents within the living system unlike the resazurin dye which is affected by all the reducing agents present in the cell and the color is easily bleached out due to prolonged incubation time, environmental $\mathrm{pH}$, and temperature $[8,10,12]$. Also, resazurin may not accurately show cellular proliferation rates because of the non-linear correlation of the dye reduction and cell density which may be more useful in screening for cytotoxicity than assessing cell proliferation [12].

Cellular based apoptosis assay. For the apoptosis methods, flow cytometry using annexin $\mathrm{V}$ indicated both early and late apoptotic rates of the cell line (Fig. 3); while the caspase 3/7 assay indicated the total cell apoptosis through Relative Light Unit (RLU) proportioned to its caspase activity (Fig. 4). Highest apoptotic rate for DOX-treated cells using flow cytometry and caspase assay were observed at concentrations $50 \mu \mathrm{g} / \mathrm{mL}$ and $25 \mu \mathrm{g} / \mathrm{mL}$, respectively. It is assumed that the reading at
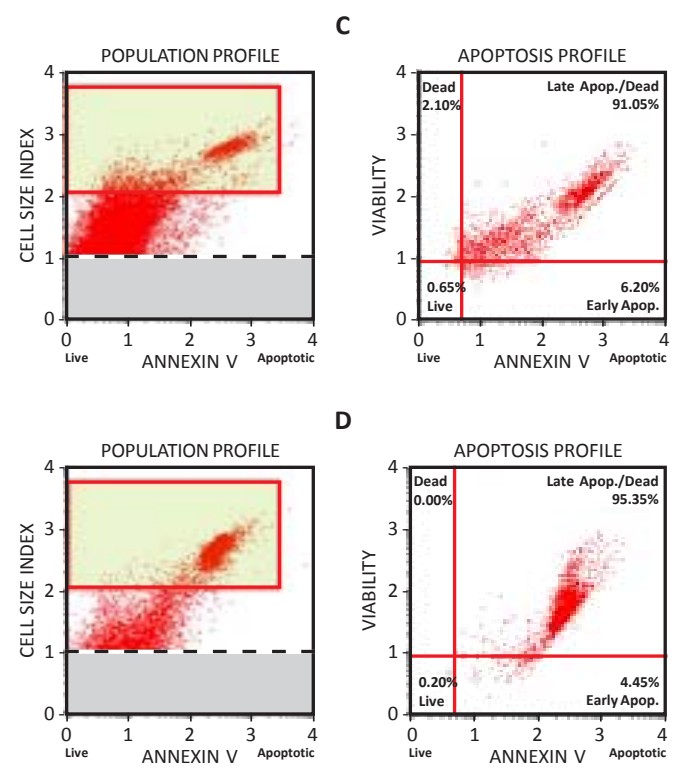

Figure 3. Apoptosis profile of colorectal adenocarcinoma cells that were (A) untreated, (B) treated with $12.5 \mu \mathrm{g} /$ $\mathrm{mL}$ DOX, (C) treated with $25 \mu \mathrm{g} / \mathrm{mL}$ DOX, and (D) treated with $50 \mu \mathrm{g} / \mathrm{mL}$ DOX. 
Tiongson TA, Magsumbol MC, Devanadera MK, \& Santiago MR | Acta Manilana 64 (2016)

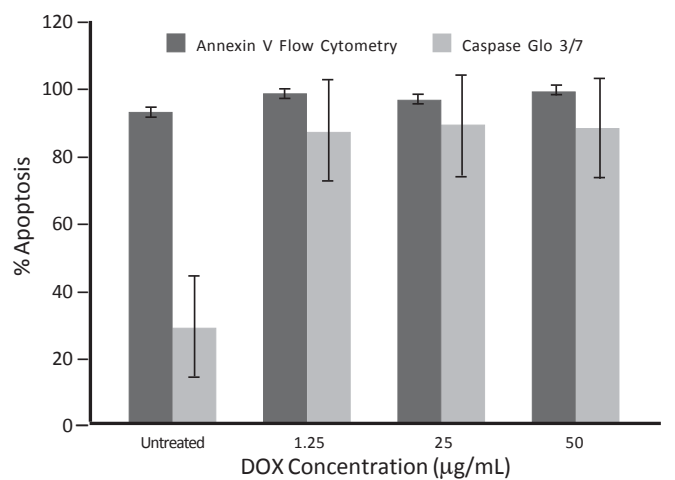

Figure 4. Apoptosis profile of colorectal adenocarcinoma cells treated with free DOX at different concentrations.

the highest concentration via caspase assay shifted its apoptotic rate downward was because the cell could have started undergoing cell death, causing it to decrease its caspase level activity, hence, decreasing also its RLU or apoptotic rate.

As seen in Table 2, both caspase 3/7 and flow cytometry are not significantly correlated in terms of apoptotic rate. Caspase 3/7 assay is used as a method of detecting apoptotic activity because of the proteins role in apoptosis pathway. Caspase-3 has been identified as one of the proteins that trigger apoptosis on the upstream part of the cascade system while caspase-7 was detected to be active in promoting apoptosis on the downstream part of the cascade system. Both proteins were used to critically detect the occurring apoptosis on the cell system upon lysis has also seen in Fig. 1. Using caspase 3 and 7 were very specific and sensitive because it uses enzyme-substrate reaction and liberated caspase in the solution from the lyse cells were easily detected due to the liberation of fluorescent substance. However, limitations on the use of caspase 3/7 have to be noted such as the substrate was temperature sensitive; substrate should be immediately used after reconstitution as it slowly loses its activity if not preserved properly and
Table 2. Comparison of Paired $t$-Test of Annexin V Flow Cytometry and Caspase

\begin{tabular}{c|c|c|c}
\hline \multicolumn{4}{c}{ Paired Samples Correlations } \\
\hline Pair 1 & $\begin{array}{c}\text { Flow Cytometry } \\
\text { and Caspase }\end{array}$ & .921 & .079 \\
\hline
\end{tabular}

detection of activity vary since the reaction is cell density dependent [12].

Annexin V used to detect phospholipids specifically the phosphatidylserine that can be found on the cell membrane. Assay procedure using annexin $\mathrm{V}$ was very sensitive since it detects early apoptosis when the cell membrane is slowly disintegrating, late apoptosis when the cell membrane is completely disintegrated, and discrimination of necrotic cell from apoptotic cell $[13,14]$. From the results obtained, it is evident that flow cytometry was the more reliable method to use for the apoptotic profile as it showed stages of cell apoptosis when treated with DOX at different concentrations.

\section{REFERENCES}

[1] Stoddart M. Cell Viability Assays: Introduction. Methods in Molecular and Cellular Biology 2011; 740:1-2.

[2] Borra RC, Lotufo MA, Gagioti SM, Barros FDM, \& Andrade PM. A Simple Method to Measure Cell Viability in Proliferation and Cytotoxicity Assays. Brazilian Oral Research 2009; 23(3):255-262.

[3] Elmore S. Apoptosis: A Review of Programmed Cell Death. Toxicologic Pathology 2007; 35(4):495-516.

[4] Goncalves M, Figueira P, Maciel D, Rodrigues J, Shi X, Tomas H, \& Li Y. Antitumor Efficacy of Doxorubicin-loaded Laponite/Alginate Hybrid Hydrogels. Macromolecular Bioscience 2014; 14(1):110-120.

[5] Trebunova M, Laputkova G, Slaba E, Lacjakova K, \& Verebova A. Effects of Docetaxel, Doxorubicin and Cyclophosphamide on Human Breast Cancer Cell Line MCF-7. Anticancer Research 2012; 32:2849-2854. 
[6] Manchun S, Dassc CR, Cheewatanakornkoola $\mathrm{K}$, \& Sriamornsaka P. Enhanced Anti-Tumor Effect of pH-responsive Rextrin Nanogels Delivering Doxorubicin on Colorectal Cancer. Carbohydrate Polymers 2015; 126:222-230.

[7] Riss TL, Moravec RA, Niles AL, Benink HA, Worzella TJ, Minor L, Storts D, \& Reid Y. Cell Viability Assays. In: Sittampalam G, Coussens N, \& Nelson H (Eds.) Assay Guidance Manual, pp. 1-23. (2013).

[8] Perrot S, Dutertre-Catella H, Martin C, Warnet JM, \& Rat P. A New Nondestructive Cytometric Assay Based on Resazurin Metabolism and an Organ Culture Model for Assessment of Corneal Viability. Cytometery Part A. 2003; 55A:7-14.

[9] Mosmann T. Rapid Colorimetric Assay for Cellular Growth and Survival: Application to Proliferation and Cytotoxicity Assays. Journal of Immunological Methods 1983; 65:55-63.

[10] Wang P, Henning S, \& Heber D. Limitations of MTT and MTS-Based Assays for Measurement of Antiproliferative Activity of Green Tea Polyphenols. PLOS ONE. 2010; 5(4):1-10.
[11] O'Brien J, Wilson I, Orton T, \& Pognan F. Investigation of the Alamar Blue (Resazurin) Fluorescent Dye for the Assessment of Mammalian Cell Cytotoxicity. European Journal of Biochemistry 2000; 267(17):5421-5426.

[12] Rampersad SN. Multiple Applications of Alamar Blue as an Indicator of Metabolic Function and Cellular Health in Cell Viability Bioassays. Sensors 2012; 12:12347-12360.

[13] Shutte B, Nuydens R, Geerts H, \& Ramaekers F. Annexin $\mathrm{V}$ Binding Assay as a Tool to Measure Apoptosis in Differentiated Neuronal Cells. Journal of Neuroscience Methods 1998; 86: 6369.

[14] Rieger AM, Nelson KL, Konowalchuck JD, \& Barreda DR. Modified Annexin V/Propidium lodide Apoptosis Assay for Accurate Assessment of Cell Death. Journal of Visualized Experiments 2011; 50: e2597. 